\title{
Wiener Index of Degree Splitting Graph of some Hydrocarbons
}

\author{
K. Thilakam \\ $P G$ and Research Department of Mathematics \\ Seethalakshmi Ramaswami College \\ Tiruchirappalli - 2
}

\author{
A. Sumathi \\ Department of Mathematics \\ Seethalakshmi Ramaswami College \\ Tiruchirappalli - 2
}

\begin{abstract}
In quantum chemistry, the physico-chemical properties of chemical compounds are often modeled by means of molecular-graph-based structure-descriptors, which are also referred to as topological indices. One of the most widely known topological descriptors is Wiener index. It is named after chemist Harold Wiener who introduced in the year 1947. It is defined by the sum of the distances between all (ordered) pairs of vertices of $\mathrm{G}$. In this paper, we find the Wiener index of degree splitting of some aliphatic and aromatic hydrocarbons and classify its characterization using MATLAB
\end{abstract}

\section{Keywords}

Hydro Carbons, Wiener Index, MATLAB

2010 Mathematics subject classification: $05 \mathrm{C} 12$, $05 \mathrm{C} 85$

\section{INTRODUCTION}

The Wiener index $\mathbf{W}(\mathbf{G})$ is a distance-based topological invariant much used in the study of the structure-property and the structure-activity relationships of various classes of biochemically interesting compounds introduced by Harold Wiener in 1947 for predicting boiling points of alkanes based on the formula

$$
b \cdot p=\alpha W+\beta w(3)+\gamma
$$

Where $\alpha, \beta, \gamma$ are empirical constants, and $\mathrm{w}(3)$ is called path number. [9]

It is defined as the half sum of the distances between all pairs of vertices of $\mathrm{G}$.

$$
W(G)=\frac{1}{2} \sum_{u, v \in G} d(u, v)
$$

Where $d(u, v)$ is the number of edges in a shortest path connecting the vertices $\mathrm{u} \& \mathrm{v}$ in $\mathrm{G} .[10]$

\section{Notation:}

$W(G)=\frac{1}{2} \sum_{u, v \in G} d(u, v)=\sum_{u<v} d(u, v)=\sum_{i<j} d\left(u_{i}, u_{j}\right)$

Suppose chemical reaction is represented as the transformation of the chemical graph representing the The calculation of HMO total $\pi$-energy (that is, $E$ ) is not an easy task. The numerical work of this paper is based on [8]. The eigenvalues of the adjacency matrix of $G$ are usually called to be the eigenvalues of G Let $\lambda_{1}, . ., \lambda_{n}$ be the eigen values of $G$ then Energy of the graph $G$ is defined by reaction's substrate into another chemical graph representing the degree splitting, the graph obtained in this manner may or may not exist in reality, but it is in the interest of the chemist to check the characterization of the so obtained new molecular structure. In this paper, we characterize the resultant degree splitting of some hydro carbons. The Wiener index $\mathrm{W}$ and the spectral radius $\lambda_{1}$ are popular topological indices. Finding the relation between $\mathrm{W}$ and $\lambda_{1}$ are very much used for modeling of properties of many types of chemical compounds. The vast majority of molecules of interest in chemistry are cyclic. Therefore finding relation between $\mathrm{W}$ and $\lambda_{1}$ is important not only in the case of alkanes, but also for other classes of organic compounds. The structural dependence of $W$ and (separately) of $\lambda_{1}$ of benzenoid hydrocarbons was studied previously. In 2008, Slavko Radenković S and Ivan Gutman have discussed the relation between $W$ and $\lambda_{1}$ for some alkanes and benzenoid molecules.[3], [7]

\section{DEFINITIONS AND PRELIMINARIES}

Our notation is standard and mainly taken from standard books of graph theory. We consider finite, nontrivial, simple and undirected graphs only. For a graph $\mathrm{G}$, we denote by $\mathrm{V}(\mathrm{G})$ and $E(G)$, its vertex and edge sets, respectively. Degree splitting graph DS(G) was introduced by R. Ponraj and S. Somasundaram. Let $G=(V ; E)$ be a graph with $V=S_{1} \cup S_{2}$ $\cup \ldots \ldots \ldots \cup \mathrm{S}_{\mathrm{t}} \cup \mathrm{T}$, where each $\mathrm{S}_{\mathrm{i}}$ is a set of vertices having at least two vertices and having the same degree and $\mathrm{T}=\mathrm{V}-\mathrm{U}$ $\mathrm{S}_{\mathrm{i}}$. The degree splitting graph of $\mathrm{G}$ denoted by $\mathrm{DS}(\mathrm{G})$ is obtained from $\mathrm{G}$ by adding vertices $\mathrm{w}_{1}, \mathrm{w}_{2}, \ldots \ldots, \mathrm{w}_{\mathrm{t}}$ and joining $w_{i}$ to each vertex of $S_{i}(1 \leq i \leq t)$. [4], [5], [6]

\begin{tabular}{|c|c|c|c|}
\hline $\begin{array}{l}\text { Molecular } \\
\text { Structure of } \\
\text { Propane }\end{array}$ & $\begin{array}{c}\text { Molecular } \\
\text { Graph } \\
\text { (G) }\end{array}$ & $\begin{array}{c}\text { Molecular } \\
\text { Structure of } \\
\text { Cyclo Butane }\end{array}$ & $\begin{array}{c}\text { Degree } \\
\text { splitting } \\
\text { of } G \\
\text { DS }(G)\end{array}$ \\
\hline 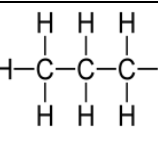 & & $\begin{array}{rl}\mathrm{H} & \mathrm{H} \\
\mathrm{I} & \mathrm{I} \\
\mathrm{H}-\mathrm{C} & \mathrm{C}-\mathrm{H} \\
\mathrm{I} & \mathrm{I} \\
\mathrm{H}-\mathrm{C} & \mathrm{C} \\
\mathrm{I} & \mathrm{I} \\
\mathrm{H} & \mathrm{H}\end{array}$ & \\
\hline
\end{tabular}

Fig.1

$\mathrm{E}(\mathrm{G})=\sum_{i=1}^{n}\left|\lambda_{i}\right|$. A graph $\mathrm{G}$ on $\mathrm{n}$ vertices is said to be hyper energetic if $E(G)>2 n-2$ and hypo energetic if $E(G)<n$. 
The largest eigenvalue of $\mathrm{G}$, referred to as the spectral radius of $\mathrm{G}$, will be labeled by $\lambda_{1}$. Using $\lambda_{1}$ as a measure of branching was proposed by one of the present authors as early as in 1977. For decades this branching index has not attracted much attention of theoretical chemists. Recently, studies of the spectral radius as the measure of branching became attractive again. W depends on the size (number of carbon atoms) of the molecules examined, it is purposeful to restrict the consideration to classes of alkane isomers. Our investigation of the relation between $\mathrm{W}$ and $\lambda_{1}$ is being performed on certain class of degree splitting molecular graphs with the respective molecular graph.

In general, an Organic compound containing only carbon and hydrogen is called hydro carbons. Hydrocarbons without benzene rings and with benzene rings are called aliphatic and aromatic hydrocarbons respectively. Aliphatic hydrocarbons are classified into acyclic and cyclic hydrocarbons. In chemical graph theory, an acyclic hydrocarbon means that chemical tree. (ie) A chemical tree is tree in which no vertex has more than four neighbours.

\section{WIENER INDICES OF G AND DS(G) FOR SOME ALIPHATIC HYDROCARBONS \\ 3.1. Comparative study between Wiener indices of $G$ and DS ( $G)$ for acyclic hydrocarbons}

The results obtained for the Wiener index of $G$ and Wiener index of degree splitting of $\mathrm{G}$ are given in Table 1 . We have calculated and tabulated the Wiener indices, Spectral radius, Energy level (for both G and DS(G)) for acyclic hydro carbons.

The Wiener index of the molecular graph $\operatorname{DS}(G)$ of the 32 acyclic hydrocarbons with $E, \lambda_{1}$

Table 1

\begin{tabular}{|c|c|c|c|c|c|c|c|}
\hline $\begin{array}{c}\begin{array}{c}\text { No. of carbon } \\
\text { atoms }\end{array} \\
\end{array}$ & $\begin{array}{c}\text { Hydro } \\
\text { Carbon } \\
\end{array}$ & $\mathbf{W}(\mathbf{G})$ & $\begin{array}{c}\text { Energy Level of } \\
\text { W(G) }\end{array}$ & \begin{tabular}{|c|}
$\begin{array}{c}\text { Spectral Radius } \\
\text { of }(\mathbf{G})\end{array}$ \\
\end{tabular} & W(DS(G)) & $\begin{array}{c}\begin{array}{c}\text { Energy Level of } \\
\text { W(DS(G)) }\end{array} \\
\end{array}$ & \begin{tabular}{|c|}
$\begin{array}{c}\text { Spectral Radius of } \\
\text { DS }(G)\end{array}$ \\
\end{tabular} \\
\hline 1 & Methane & - & & & - & - & - \\
\hline 2 & Ethane & 1 & $\begin{array}{l}\text { Non Hyper energetic } \\
\text { Non Hypo energetic }\end{array}$ & 1.0 & 3 & $\begin{array}{c}\text { Hyper energetic } \\
\text { Non Hypo energetic }\end{array}$ & 2.0 \\
\hline 3 & Propane & 4 & $\begin{array}{c}\text { Non Hyper energetic } \\
\text { Hypo energetic }\end{array}$ & 1.40 & 8 & $\begin{array}{l}\text { Non Hyper energetic } \\
\text { Non Hypo energetic }\end{array}$ & 2.0 \\
\hline 4 & Butane & 10 & $\begin{array}{l}\text { Non Hyper energetic } \\
\text { Non Hypo energetic }\end{array}$ & 1.61 & 24 & $\begin{array}{c}\text { Hyper energetic } \\
\text { Non Hypo energetic }\end{array}$ & 2.43 \\
\hline 4 & 2-Methylpropane & 9 & $\begin{array}{c}\text { Non Hyper energetic } \\
\text { Hypo energetic }\end{array}$ & 1.73 & 14 & $\begin{array}{c}\text { Non Hyper energetic } \\
\text { Hypo energetic }\end{array}$ & 2.44 \\
\hline 5 & Pentane & 20 & $\begin{array}{l}\text { Non Hyper energetic } \\
\text { Non Hypo energetic }\end{array}$ & 1.73 & 37 & $\begin{array}{c}\text { Hyper energetic } \\
\text { Non Hypo energetic } \\
\end{array}$ & 2.76 \\
\hline 5 & 2-Methylbutane & 18 & $\begin{array}{l}\text { Non Hyper energetic } \\
\text { Non Hypo energetic }\end{array}$ & 1.8 & 23 & $\begin{array}{l}\text { Non Hyper energetic } \\
\text { Non Hypo energetic }\end{array}$ & 2.39 \\
\hline 5 & 2.2-Dimethylpropane & 16 & $\begin{array}{c}\text { Hyper energetic } \\
\text { Non Hypo energetic }\end{array}$ & 2.00 & 22 & $\begin{array}{c}\text { Non Hyper energetic } \\
\text { Hypo energetic }\end{array}$ & 2.82 \\
\hline 6 & Hexane & 35 & $\begin{array}{c}\text { Hyper energetic } \\
\text { Non Hypo energetic }\end{array}$ & 1.80 & 52 & $\begin{array}{c}\text { Hyper energetic } \\
\text { Non Hypo energetic }\end{array}$ & 3.04 \\
\hline 6 & 2-Methylpentane & 32 & $\begin{array}{l}\text { Non Hyper energetic } \\
\text { Non Hypo energetic }\end{array}$ & 1.90 & 53 & $\begin{array}{l}\text { Non Hyper energetic } \\
\text { Non Hypo energetic }\end{array}$ & 2.57 \\
\hline 6 & 3-Methylpentane & 31 & $\begin{array}{l}\text { Non Hyper energetic } \\
\text { Non Hypo energetic }\end{array}$ & 1.93 & 50 & $\begin{array}{l}\text { Non Hyper energetic } \\
\text { Non Hypo energetic }\end{array}$ & 2.56 \\
\hline 6 & 2,2-Dimethylbutane & 28 & $\begin{array}{c}\text { Non Hyper energetic } \\
\text { Hypo energetic }\end{array}$ & 2.07 & 33 & $\begin{array}{l}\text { Non Hyper energetic } \\
\text { Non Hypo energetic }\end{array}$ & 2.75 \\
\hline 6 & 2,3-Dimethylbutane & 29 & $\begin{array}{l}\text { Non Hyper energetic } \\
\text { Non Hypo energetic }\end{array}$ & 2.00 & 46 & $\begin{array}{l}\text { Non Hyper energetic } \\
\text { Non Hypo energetic }\end{array}$ & 2.80 \\
\hline 7 & Heptane & 56 & $\begin{array}{l}\text { Non Hyper energetic } \\
\text { Non Hypo energetic }\end{array}$ & 1.87 & 70 & $\begin{array}{c}\text { Hyper energetic } \\
\text { Non Hypo energetic }\end{array}$ & 3.29 \\
\hline 7 & 2-Methylhexane & 52 & $\begin{array}{l}\text { Non Hyper energetic } \\
\text { Non Hypo energetic }\end{array}$ & 1.93 & 72 & $\begin{array}{l}\text { Non Hyper energetic } \\
\text { Non Hypo energetic }\end{array}$ & 2.80 \\
\hline 7 & 3-Methylhexane & 50 & $\begin{array}{l}\text { Non Hyper energetic } \\
\text { Non Hypo energetic }\end{array}$ & 1.96 & 54 & $\begin{array}{l}\text { Non Hyper energetic } \\
\text { Non Hypo energetic }\end{array}$ & 2.31 \\
\hline 7 & 2,2-Dimethylpentane & 46 & $\begin{array}{c}\text { Non Hyper energetic } \\
\text { Hypo energetic }\end{array}$ & 2.10 & 69 & $\begin{array}{l}\text { Non Hyper energetic } \\
\text { Non Hypo energetic }\end{array}$ & 2.81 \\
\hline 7 & 2,3-Dimethylpentane & 46 & $\begin{array}{l}\text { Non Hyper energetic } \\
\text { Non Hypo energetic }\end{array}$ & 2.05 & 65 & $\begin{array}{l}\text { Non Hyper energetic } \\
\text { Non Hypo energetic }\end{array}$ & 2.88 \\
\hline 7 & 2,4-Dimethylpentane & 48 & $\begin{array}{c}\text { Non Hyper energetic } \\
\text { Hypo energetic }\end{array}$ & 2.00 & 66 & $\begin{array}{l}\text { Non Hyper energetic } \\
\text { Non Hypo energetic }\end{array}$ & 2.82 \\
\hline
\end{tabular}




\begin{tabular}{|c|c|c|c|c|c|c|c|}
\hline 7 & 3,3-Dimethylpentane & 44 & $\begin{array}{l}\text { Non Hyper energetic } \\
\text { Non Hypo energetic }\end{array}$ & 2.13 & 65 & $\begin{array}{l}\text { Non Hyper energetic } \\
\text { Non Hypo energetic }\end{array}$ & 2.82 \\
\hline 7 & 3 Ethylpentane & 48 & $\begin{array}{l}\text { Non Hyper energetic } \\
\text { Non Hypo energetic }\end{array}$ & 2.00 & 72 & $\begin{array}{l}\text { Non Hyper energetic } \\
\text { Non Hypo energetic }\end{array}$ & 2.5 \\
\hline 7 & 2,2,3-Trimethebutane & 42 & $\begin{array}{l}\text { Non Hyper energetic } \\
\text { Hypo energetic }\end{array}$ & 2.17 & 45 & $\begin{array}{l}\text { Non Hyper energetic } \\
\text { Non Hypo energetic }\end{array}$ & 2.97 \\
\hline 8 & Octane & 84 & $\begin{array}{l}\text { Non Hyper energetic } \\
\text { Non Hypo energetic }\end{array}$ & 1.87 & 90 & $\begin{array}{c}\text { Hyper energetic } \\
\text { Non Hypo energetic }\end{array}$ & 3.51 \\
\hline 8 & $\begin{array}{c}2,2,3,3- \\
\text { Trimethylbutane }\end{array}$ & 58 & $\begin{array}{l}\text { Non Hyper energetic } \\
\text { Hypo energetic }\end{array}$ & 2.30 & 76 & $\begin{array}{l}\text { Non Hyper energetic } \\
\text { Non Hypo energetic }\end{array}$ & 3.40 \\
\hline 8 & $\begin{array}{l}\text { 3-Ethyl-2- } \\
\text { methylpentane }\end{array}$ & 67 & $\begin{array}{l}\text { Non Hyper energetic } \\
\text { Non Hypo energetic }\end{array}$ & 2.92 & 114 & $\begin{array}{l}\text { Non Hyper energetic } \\
\text { Non Hypo energetic }\end{array}$ & 2.92 \\
\hline 8 & 3-Ethylhexane & 72 & $\begin{array}{l}\text { Non Hyper energetic } \\
\text { Non Hypo energetic }\end{array}$ & 2.02 & 88 & $\begin{array}{l}\text { Non Hyper energetic } \\
\text { Non Hypo energetic }\end{array}$ & 2.98 \\
\hline 8 & 3-Methylheptane & 76 & $\begin{array}{l}\text { Non Hyper energetic } \\
\text { Non Hypo energetic }\end{array}$ & 1.99 & 89 & $\begin{array}{l}\text { Non Hyper energetic } \\
\text { Non Hypo energetic }\end{array}$ & 3.00 \\
\hline 8 & 4-Methylheptane & 75 & $\begin{array}{l}\text { Non Hyper energetic } \\
\text { Non Hypo energetic }\end{array}$ & 2.00 & 88 & $\begin{array}{l}\text { Non Hyper energetic } \\
\text { Non Hypo energetic }\end{array}$ & 2.99 \\
\hline 8 & 2,2-Dimethylhexane & 71 & $\begin{array}{l}\text { Non Hyper energetic } \\
\text { Non Hypo energetic }\end{array}$ & 2.92 & 91 & $\begin{array}{l}\text { Non Hyper energetic } \\
\text { Non Hypo energetic }\end{array}$ & 2.92 \\
\hline 8 & 2,3-Dimethylhexane & 70 & $\begin{array}{l}\text { Non Hyper energetic } \\
\text { Non Hypo energetic }\end{array}$ & 2.92 & 116 & $\begin{array}{l}\text { Non Hyper energetic } \\
\text { Non Hypo energetic }\end{array}$ & 2.92 \\
\hline 8 & 2,4-Dimethylhexane & 71 & $\begin{array}{l}\text { Non Hyper energetic } \\
\text { Non Hypo energetic }\end{array}$ & 2.04 & 111 & $\begin{array}{l}\text { Non Hyper energetic } \\
\text { Non Hypo energetic }\end{array}$ & 2.89 \\
\hline 8 & 2,5-Dimethylhexane & 74 & $\begin{array}{l}\text { Non Hyper energetic } \\
\text { Non Hypo energetic }\end{array}$ & 2.00 & 113 & $\begin{array}{l}\text { Non Hyper energetic } \\
\text { Non Hypo energetic }\end{array}$ & 2.87 \\
\hline
\end{tabular}

We deem that these data will suffice for practically all imaginable considerations in chemical graph theory. The most remarkable feature seen in Table-1 is the fact that degree splitting of the acyclic Hydrocarbons will be cyclic and spectral radius of $G \& D S(G)$ variation increases maximum by one. The practical importance of this finding is that almost all degree splitting of acyclic molecules are Non Hypo energetic whatever G is Hypo energetic or not.

\section{WIENER INDICES OF DEGREE SPLITTING OF SOME POLYCYCLIC AROMATIC HYDROCARBONS}

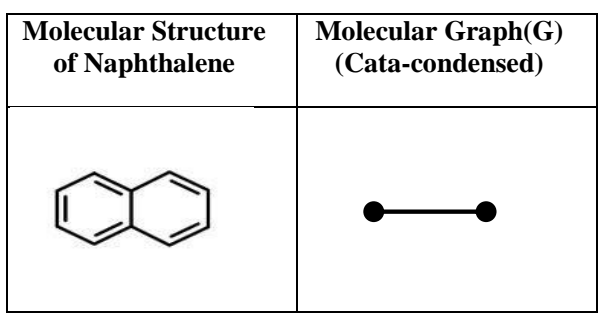

In this section, we analyze the characterization between poly cyclic aromatic benzenoid hydrocarbons and degree splitting of some poly cyclic aromatic benzenoid hydrocarbons (both catacondensed, not cata condensed). The Characteristic graph of a given benzenoid graph(cyclic graph) consists of vertices corresponding to hexagon(cycle) of the graph; two vertices are adjacent if the corresponding hexagons (cycle) share an edge. The benzenoid graph (cyclic) is called Cata-condensed if its Characteristic graph is a tree.[1], [2] To analyze the characterization between the molecules, we have taken the sample set of 12 poly cyclic benzenoid molecules (6 cata condensed, 6 not cata condensed).

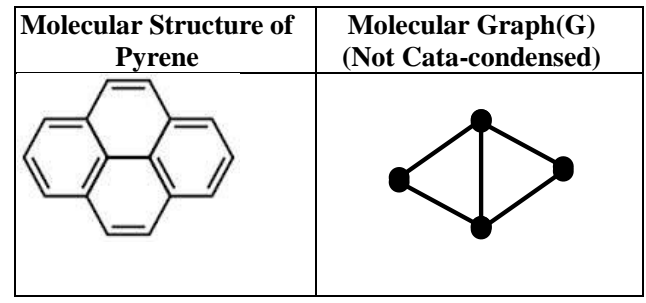

Fig.3

Fig.2 


\subsection{Wiener Indices of Degree Splitting of Some Poly Cyclic Aromatic Hydrocarbons (CATA- Condensed)}

The results obtained for the Wiener index of $\mathrm{G}$ and Wiener index of degree splitting of $G$ are given in Table 2. In additional, we tabulated the Spectral radius, Energy level (for both $\mathrm{G}$ and $\mathrm{DS}(\mathrm{G})$ ) for polycyclic (cata condensed) aromatic hydrocarbons

The Wiener index of the DS(G) of the 6 polycyclic aromatic hydrocarbons (Cata-condensed) with E, $\lambda_{1}$

Table 2

\begin{tabular}{|c|c|c|c|c|c|c|c|}
\hline $\begin{array}{c}\text { No. of carbon } \\
\text { atoms }\end{array}$ & $\begin{array}{c}\text { Hydro } \\
\text { Carbon }\end{array}$ & $\mathbf{W}(\mathbf{G})$ & Energy Level of W(G) & $\begin{array}{c}\text { Spectral } \\
\text { Radius of } \\
(\mathbf{G})\end{array}$ & $\mathbf{W}(\mathbf{D S}(\mathbf{G})$ & $\begin{array}{c}\text { Energy Level of } \\
\text { W(DS(G)) }\end{array}$ & $\begin{array}{c}\text { Spectral } \\
\text { Radius of } \\
\text { DS(G) }\end{array}$ \\
\hline 12 & Biiphenylene & 180 & $\begin{array}{c}\text { Non Hyper energetic } \\
\text { Non Hypo energetic }\end{array}$ & 2.5321 & 177 & $\begin{array}{c}\text { Hyper energetic } \\
\text { Non Hypo energetic }\end{array}$ & 4.0000 \\
\hline 13 & Fluorene & 219 & $\begin{array}{l}\text { Non Hyper energetic } \\
\text { Non Hypo energetic }\end{array}$ & 2.4687 & 205 & $\begin{array}{c}\text { Hyper energetic } \\
\text { Non Hypo energetic }\end{array}$ & 4.1227 \\
\hline 18 & Napththacene & 633 & $\begin{array}{c}\text { Non Hyper energetic } \\
\text { Non Hypo energetic }\end{array}$ & 2.3007 & 400 & $\begin{array}{c}\text { Non Hyper energetic } \\
\text { Non Hypo energetic }\end{array}$ & 4.4260 \\
\hline 24 & Tetraphenylene & 1080 & $\begin{array}{l}\text { Non Hyper energetic } \\
\text { Non Hypo energetic }\end{array}$ & 2.5321 & 703 & $\begin{array}{c}\text { Non Hyper energetic } \\
\text { Non Hypo energetic }\end{array}$ & 5.0314 \\
\hline 26 & Hexacene & 1637 & $\begin{array}{l}\text { Non Hyper energetic } \\
\text { Non Hypo energetic }\end{array}$ & 2.5129 & 820 & $\begin{array}{c}\text { Non Hyper energetic } \\
\text { Non Hypo energetic }\end{array}$ & 5.0817 \\
\hline 30 & Heptacene & 2479 & $\begin{array}{l}\text { Non Hyper energetic } \\
\text { Non Hypo energetic }\end{array}$ & 2.5243 & 1095 & $\begin{array}{c}\text { Non Hyper energetic } \\
\text { Non Hypo energetic }\end{array}$ & 5.3288 \\
\hline
\end{tabular}

\subsection{Wiener Indices of Degree Splitting of Poly Cyclic Aromatic Hydrocarbons (Not \\ CATA-Condensed)}

Here, we have tabulated the Wiener index, Spectral radius, Energy level (for both G and DS(G)) for polycyclic (not cata condensed) aromatic hydrocarbons.

The Wiener index of the DS(G) of the 6 polycyclic aromatic hydrocarbons (Not Cata-condensed) with $E$, $\lambda_{1}$

Table3.

\begin{tabular}{|c|l|l|l|l|l|c|c|}
\hline $\begin{array}{c}\text { No. of carbon } \\
\text { Atoms }\end{array}$ & $\begin{array}{c}\text { Hydro } \\
\text { Carbon }\end{array}$ & $\mathbf{W}(\mathbf{G})$ & $\begin{array}{c}\text { Energy Level of } \\
\mathbf{W}(\mathbf{G})\end{array}$ & $\begin{array}{c}\text { Spectral } \\
\text { Radius of } \\
(\mathbf{G})\end{array}$ & $\begin{array}{c}\text { W(DS( } \\
\mathbf{G}))\end{array}$ & $\begin{array}{c}\text { Energy Level of } \\
\text { W(DS(G)) }\end{array}$ & $\begin{array}{c}\text { Spectral Radius of } \\
\text { DS(G) }\end{array}$ \\
\hline 12 & Acenaphthene & 166 & $\begin{array}{c}\text { Non Hyper energetic } \\
\text { Non Hypo energetic }\end{array}$ & 2.4708 & 174 & $\begin{array}{c}\text { Hyper energetic } \\
\text { Non Hypo energetic }\end{array}$ & 4.0000 \\
\hline 13 & Phenalene & 210 & $\begin{array}{c}\text { Non Hyper energetic } \\
\text { Non Hypo energetic }\end{array}$ & 2.4495 & 205 & $\begin{array}{c}\text { Hyper energetic } \\
\text { Non Hypo energetic }\end{array}$ & 4.1226 \\
\hline 18 & Pleiadene & 519 & $\begin{array}{l}\text { Non Hyper energetic } \\
\text { Non Hypo energetic }\end{array}$ & 2.4815 & 395 & $\begin{array}{c}\text { Hyper energetic } \\
\text { Non Hypo energetic }\end{array}$ & 4.5443 \\
\hline 24 & Coronene & 1002 & $\begin{array}{c}\text { Non Hyper energetic } \\
\text { Non Hypo energetic }\end{array}$ & 2.6751 & 721 & $\begin{array}{c}\text { Non Hyper energetic } \\
\text { Non Hypo energetic }\end{array}$ & 5.0000 \\
\hline 30 & Rubicene & 1314 & $\begin{array}{l}\text { Non Hyper energetic } \\
\text { Non Hypo energetic }\end{array}$ & 2.6153 & 863 & $\begin{array}{c}\text { Non Hyper energetic } \\
\text { Non Hypo energetic }\end{array}$ & 5.0651 \\
\hline
\end{tabular}

From 4.1 and 4.2., we see that there is no much difference of the topological index $\lambda_{1}$ of degree splitting of polycyclic aromatic hydrocarbons between Cata-condensed and not Catacondensed. But topological index $\lambda_{1}$ of degree splitting of polycyclic aromatic hydrocarbons is twice as $\lambda_{1}$ of polycyclic aromatic hydrocarbons (Cata-condensed and not Cata- condensed). Energy level of polycyclic aromatic hydrocarbons (both Cata-condensed and not Cata-condensed) and degree splitting of polycyclic aromatic hydrocarbons (both Catacondensed and not Cata-condensed) are non hypo energetic. But Energy level of some degree splitting of polycyclic aromatic hydrocarbons (both Cata-condensed and not Catacondensed) becomes hyper energetic. 


\section{CONCLUSION}

In this paper, we have examined the Wiener index of degree splitting of various hydro carbons and have classified its characterization like Energy, Spectral radius with hydro carbons using MATLAB.

\section{REFERENCES}

[1] Andrey A. Dobrynin, Congruence Relations for the Wiener index of Hexagonal Chains, J. chem. Inf. Comput. Sci 1997, 37,1109-1110

[2] Andrey A. Dobrynin, New congruence Relations for Wiener index of Cata-Condensed Benzenoid Graphs, J. chem. Inf. Comput. Sci 1998, 38,405-409.

[3] Ivan Gutman and Drago`s Cvetkovi'c, Selected Topics on Applications of Graph Spectra, ISBN 978-86-8059344-9 (2011)

[4] Ponraj R and Somasundaram S, On the degree splitting graph of a graph, NATL ACAD,SCI LETT, 27(7\& 8) (2004), 275-278.
[5] Sampathkumar E. and Walikar H.B., On Splitting Graph of a Graph, J. Karnatak Univ. Sci.,25(13) (1980), 13-16.

[6] Santhi Maheswari N. R. and Sekar C, On the d2splitting graph of a graph, Kragujevac Journal of Mathematics, Volume 36 Number 2 (2012), Pages 315 321

[7] Slavko Radenković S and Ivan Gutman, Relation between Wiener index and spectral radius, Kragujevac J. Sci. $30 \quad$ (2008) 57-64

[8] Thilakam K, Sumathi A, How to Compute the Wiener index of a graph using MATLAB, International Journal of Applied Mathematics\& Statistical Sciences (IJAMSS), ISSN(P): 2319-3972;,Vol. 2, Issue 5, Nov 2013, 143148.

[9] Wiener H, Structural determination of paraffin boiling points- J.Am chem. Soc.6 (1947) 17-20

[10] http://mathworld.wolfram.com/WienerIndex 